\title{
Global Dynamics of Certain Homogeneous Second-Order Quadratic Fractional Difference Equation
}

\author{
M. Garić-Demirović, ${ }^{1}$ M. R. S. Kulenović, ${ }^{2}$ and M. Nurkanović ${ }^{1}$ \\ ${ }^{1}$ Department of Mathematics, University of Tuzla, 75000 Tuzla, Bosnia and Herzegovina \\ ${ }^{2}$ Department of Mathematics, University of Rhode Island, Kingston, RI 02881-0816, USA
}

Correspondence should be addressed to M. R. S. Kulenović; kulenm@math.uri.edu

Received 31 August 2013; Accepted 1 October 2013

Academic Editors: H. Iiduka and S. A. Mohiuddine

Copyright (C) 2013 M. Garić-Demirović et al. This is an open access article distributed under the Creative Commons Attribution License, which permits unrestricted use, distribution, and reproduction in any medium, provided the original work is properly cited.

We investigate the basins of attraction of equilibrium points and minimal period-two solutions of the difference equation of the form $x_{n+1}=x_{n-1}^{2} /\left(a x_{n}^{2}+b x_{n} x_{n-1}+c x_{n-1}^{2}\right), n=0,1,2, \ldots$, where the parameters $a, b$, and $c$ are positive numbers and the initial conditions $x_{-1}$ and $x_{0}$ are arbitrary nonnegative numbers. The unique feature of this equation is the coexistence of an equilibrium solution and the minimal period-two solution both of which are locally asymptotically stable.

\section{Introduction}

We investigate global behavior of the equation

$$
x_{n+1}=\frac{x_{n-1}^{2}}{a x_{n}^{2}+b x_{n} x_{n-1}+c x_{n-1}^{2}}=f\left(x_{n}, x_{n-1}\right),
$$

where the parameters $a, b$, and $c$ are positive numbers and the initial conditions $x_{-1}$, and $x_{0}$ are arbitrary nonnegative numbers such that $x_{-1}+x_{0}>0$. Equation (1) is a special case of equations

$$
\begin{aligned}
& x_{n+1}=\frac{A x_{n}^{2}+B x_{n} x_{n-1}+C x_{n-1}^{2}}{a x_{n}^{2}+b x_{n} x_{n-1}+c x_{n-1}^{2}}, \quad n=0,1,2, \ldots, \\
& x_{n+1}=\frac{A x_{n}^{2}+B x_{n} x_{n-1}+C x_{n-1}^{2}+D x_{n}+E x_{n-1}+F}{a x_{n}^{2}+b x_{n} x_{n-1}+c x_{n-1}^{2}+d x_{n}+e x_{n-1}+f}, \\
& n=0,1,2, \ldots .
\end{aligned}
$$

Some special cases of (3) have been considered in the series of papers [1-5]. Some special second-order quadratic fractional difference equations have appeared in analysis of competitive and anticompetitive systems of linear fractional difference equations in the plane; see [6-10]. Describing the global dynamics of (3) is a formidable task as this equation contains as a special case many equations with complicated dynamics, such as the linear fractional difference equation

$$
x_{n+1}=\frac{D x_{n}+E x_{n-1}+F}{d x_{n}+e x_{n-1}+f}, \quad n=0,1,2, \ldots
$$

which dynamics was investigated in great detail in [11] and in many papers which solved some conjectures and open problems posed in [11]. Equation (2) can be brought to the form

$$
x_{n+1}=\frac{A\left(x_{n} / x_{n-1}\right)^{2}+B\left(x_{n} / x_{n-1}\right)+C}{a\left(x_{n} / x_{n-1}\right)^{2}+b\left(x_{n} / x_{n-1}\right)+c}, \quad n=0,1,2, \ldots,
$$

and one can take the advantage of this auxiliary equation to describe the dynamics of (2). This approach was used in $[1-4,12]$. In this paper, we take a different approach based on the theory of monotone maps developed in $[13,14]$ and use it to describe precisely the basins of attraction of all attractors of this equation. The special case of (1) when 
$a=0$ is the linear fractional difference equation whose global dynamics is described in [11]. We show that (1) exhibits three types of global behavior characterized by the existence of a unique positive equilibrium solution and one or two minimal period-two solutions, one of which is locally stable and the other is a saddle point. The unique feature of (1) is the coexistence of an equilibrium solution and the minimal period-two solution both being locally asymptotically stable. This new phenomenon is caused by the presence of quadratic terms and did not exist in the case of (4).

Our results will be based on the following theorem for a general second-order difference equation

$$
x_{n+1}=f\left(x_{n}, x_{n-1}\right), \quad n=0,1,2, \ldots ;
$$

see $[15]$.

Theorem 1. Let $I$ be a set of real numbers and let $f: I \times$ $I \rightarrow I$ be a function which is nonincreasing in the first variable and nondecreasing in the second variable. Then, for every solution $\left\{x_{n}\right\}_{n=-1}^{\infty}$ of the equation

$$
x_{n+1}=f\left(x_{n}, x_{n-1}\right), x_{-1}, x_{0} \in I, n=0,1,2, \ldots,
$$

the subsequences $\left\{x_{2 n}\right\}_{n=0}^{\infty}$ and $\left\{x_{2 n-1}\right\}_{n=0}^{\infty}$ of even and odd terms of the solution do exactly one of the following:

(i) eventually, they are both monotonically increasing;

(ii) eventually, they are both monotonically decreasing;

(iii) one of them is monotonically increasing and the other is monotonically decreasing.

The consequence of Theorem 1 is that every bounded solution of (7) converges to either equilibrium or periodtwo solution or to the point on the boundary, and the most important question becomes determining the basins of attraction of these solutions as well as the unbounded solutions. The answer to this question follows from an application of theory of monotone maps in the plane which will be presented in Section 2.

\section{Preliminaries}

We now give some basic notions about monotone maps in the plane.

Consider a partial ordering $\preceq$ on $\mathbb{R}^{2}$. Two points $x, y \in$ $\mathbb{R}^{2}$ are said to be related if $x \preceq y$ or $y \preceq x$. Also, a strict inequality between points may be defined as $x \prec y$ if $x \prec$ $y$ and $x \neq y$. A stronger inequality may be defined as $x=$ $\left(x_{1}, x_{2}\right) \ll y=\left(y_{1}, y_{2}\right)$ if $x \preceq y$ with $x_{1} \neq y_{1}$ and $x_{2} \neq y_{2}$.

A map $T$ on a nonempty set $\mathscr{R} \subset \mathbb{R}^{2}$ is a continuous function $T: \mathscr{R} \rightarrow \mathscr{R}$. The map $T$ is monotone if $x \preceq y$ implies that $T(x) \preceq T(y)$ for all $x, y \in \mathscr{R}$, and it is strongly monotone on $\mathscr{R}$ if $x \prec y$ implies that $T(x) \ll T(y)$ for all $x, y \in \mathscr{R}$. The map is strictly monotone on $\mathscr{R}$ if $x \prec y$ implies that $T(x) \prec T(y)$ for all $x, y \in \mathscr{R}$. Clearly, being related is invariant under iteration of a strongly monotone map.

Throughout this paper, we will use the North-East ordering (NE) for which the positive cone is the first quadrant; that is, this partial ordering is defined by $\left(x_{1}, y_{1}\right) \preceq_{\mathrm{ne}}\left(x_{2}, y_{2}\right)$ if $x_{1} \leq x_{2}$ and $y_{1} \leq y_{2}$ and the SouthEast (SE) ordering defined as $\left(x_{1}, y_{1}\right) \preceq_{\mathrm{se}}\left(x_{2}, y_{2}\right)$ if $x_{1} \leq$ $x_{2}$ and $y_{1} \geq y_{2}$.

A map $T$ on a nonempty set $\mathscr{R} \subset \mathbb{R}^{2}$ which is monotone with respect to the North-East ordering is called cooperative and a map monotone with respect to the South-East ordering is called competitive.

If $T$ is a differentiable map on a nonempty set $\mathscr{R}$, a sufficient condition for $T$ to be strongly monotone with respect to the SE ordering is that the Jacobian matrix at all points $x$ has the following sign configuration:

$$
\operatorname{sign}\left(J_{T}(\mathrm{x})\right)=\left[\begin{array}{ll}
+ & - \\
- & +
\end{array}\right],
$$

provided that $\mathscr{R}$ is open and convex.

For $x \in \mathbb{R}^{2}$, define $Q_{\ell}(x)$ for $\ell=1, \ldots, 4$ to be the usual four quadrants based at $x$ and numbered in a counterclockwise direction; for example, $Q_{1}(x)=\left\{y \in R^{2}\right.$ : $\left.x_{1} \leq y_{1}, x_{2} \leq y_{2}\right\}$. Basin of attraction of a fixed point $(\bar{x}, \bar{y})$ of a map $T$, denoted as $\mathscr{B}((\bar{x}, \bar{y}))$, is defined as the set of all initial points $\left(x_{0}, y_{0}\right)$ for which the sequence of iterates $T^{n}\left(\left(x_{0}, y_{0}\right)\right)$ converges to $(\bar{x}, \bar{y})$. Similarly, we define a basin of attraction of a periodic point of period $p$. The next five results, from $[13,14]$, are useful for determining basins of attraction of fixed points of competitive maps. Related results have been obtained by Smith in $[5,16]$.

Theorem 2. Let $T$ be a competitive map on a rectangular region $\mathscr{R} \subset \mathbb{R}^{2}$. Let $\overline{\mathrm{x}} \in \mathscr{R}$ be a fixed point of $T$ such that $\Delta:=$ $\mathscr{R} \cap \operatorname{int}\left(Q_{1}(\overline{\mathrm{x}}) \cup Q_{3}(\overline{\mathrm{x}})\right.$ ) is nonempty (i.e., $\overline{\mathrm{x}}$ is not the $N W$ or $S E$ vertex of $\mathscr{R})$, and $T$ is strongly competitive on $\Delta$. Suppose that the following statements are true.

(a) The map $T$ has a $C^{1}$ extension to a neighborhood of $\overline{\mathrm{x}}$.

(b) The Jacobian $J_{T}(\overline{\mathrm{x}})$ of $T$ at $\overline{\mathrm{x}}$ has real eigenvalues $\lambda, \mu$ such that $0<|\lambda|<\mu$, where $|\lambda|<1$, and the eigenspace $E^{\lambda}$ associated with $\lambda$ is not a coordinate axis.

Then, there exists a curve $\mathscr{C} \subset \mathscr{R}$ through $\overline{\mathrm{x}}$ that is invariant and a subset of the basin of attraction of $\overline{\mathrm{x}}$, such that $\mathscr{C}$ is tangential to the eigenspace $E^{\lambda}$ at $\overline{\mathrm{x}}$, and $\mathscr{C}$ is the graph of a strictly increasing continuous function of the first coordinate on an interval. Any endpoints of $\mathscr{C}$ in the interior of $\mathscr{R}$ are either fixed points or minimal period-two points. In the latter case, the set of endpoints of $\mathscr{C}$ is a minimal period-two orbit of $T$.

We will see in Theorem 4 that the situation where the endpoints of $\mathscr{C}$ are boundary points of $\mathscr{R}$ is of interest. The following result gives a sufficient condition for this case.

Theorem 3. For the curve $\mathscr{C}$ of Theorem 2 to have endpoints in $\partial \mathscr{R}$, it is sufficient that at least one of the following conditions is satisfied.

(i) The map T has no fixed points nor periodic points of minimal period-two in $\Delta$.

(ii) The map $T$ has no fixed points in $\Delta$, $\operatorname{det} J_{T}(\overline{\mathrm{x}})>0$, and $T(x)=\overline{\mathrm{x}}$ has no solutions $x \in \Delta$. 
(iii) The map $T$ has no points of minimal period-two in $\Delta$, det $J_{T}(\overline{\mathrm{x}})<0$, and $T(x)=\overline{\mathrm{x}}$ has no solutions $x \in \Delta$.

For maps that are strongly competitive near the fixed point, hypothesis (b) of Theorem 2 reduces just to $|\lambda|<1$. This follows from a change of variables [5] that allows the Perron-Frobenius theorem to be applied. Also, one can show that in such case no associated eigenvector is aligned with a coordinate axis.

The next result is useful for determining basins of attraction of fixed points of competitive maps.

Theorem 4. (A) Assume the hypotheses of Theorem 2, and let $\mathscr{C}$ be the curve whose existence is guaranteed by Theorem 2. If the endpoints of $\mathscr{C}$ belong to $\partial \mathscr{R}$, then $\mathscr{C}$ separates $\mathscr{R}$ into two connected components, namely,

$$
\begin{aligned}
& \mathscr{W}_{-}:=\left\{x \in \mathscr{R} \backslash \mathscr{C}: \exists y \in \mathscr{C} \text { with } \mathrm{x} \preceq_{s e} y\right\}, \\
& \mathscr{W}_{+}:=\left\{x \in \mathscr{R} \backslash \mathscr{C}: \exists y \in \mathscr{C} \text { with } y \preceq_{s e} x\right\},
\end{aligned}
$$

such that the following statements are true.

(i) $\mathscr{W}_{-}$is invariant, and $\operatorname{dist}\left(T^{n}(x), Q_{2}(\overline{\mathrm{x}})\right)$ 0 as $n \rightarrow \infty$ for every $x \in \mathscr{W}_{-}$.

(ii) $\mathscr{W}_{+}$is invariant, and $\operatorname{dist}\left(T^{n}(x), Q_{4}(\overline{\mathrm{x}})\right) \quad \rightarrow$ 0 as $n \rightarrow \infty$ for every $x \in \mathscr{W}_{+}$.

(B) If, in addition to the hypotheses of part $(A), \overline{\mathrm{x}}$ is an interior point of $\mathscr{R}$ and $T$ is $C^{2}$ and strongly competitive in a neighborhood of $\bar{x}$, then $T$ has no periodic points in the boundary of $Q_{1}(\overline{\mathrm{x}}) \cup Q_{3}(\overline{\mathrm{x}})$ except for $\overline{\mathrm{x}}$, and the following statements are true.

(iii) For every $x \in \mathscr{W}_{\text {, }}$, there exists $n_{0} \in \mathbb{N}$ such that $T^{n}(x) \in \operatorname{int} Q_{2}(\bar{x})$ for $n \geq n_{0}$.

(iv) For every $x \in \mathscr{W}_{+}$, there exists $n_{0} \in \mathbb{N}$ such that $T^{n}(x) \in \operatorname{int} Q_{4}(\bar{x})$ for $n \geq n_{0}$.

If $T$ is a map on a set $\mathscr{R}$ and if $\overline{\mathrm{x}}$ is a fixed point of $T$, the stable set $\mathscr{W}^{s}(\overline{\mathrm{x}})$ of $\overline{\mathrm{x}}$ is the set $\left\{x \in \mathscr{R}: T^{n}(x) \rightarrow \overline{\mathrm{x}}\right\}$ and unstable set $\mathscr{W}^{u}(\overline{\mathrm{x}})$ of $\overline{\mathrm{x}}$ is the set

$$
\begin{aligned}
& \left\{x \in \mathscr{R} \text { : there exists }\left\{x_{n}\right\}_{n=-\infty}^{0} \subset \mathscr{R} \text { s.t. } T\left(x_{n}\right)=x_{n+1},\right. \\
& \left.\quad x_{0}=x, \lim _{n \rightarrow-\infty} x_{n}=\bar{x}\right\} .
\end{aligned}
$$

When $T$ is noninvertible, the set $\mathscr{W}^{s}(\overline{\mathrm{x}})$ may not be connected and made up of infinitely many curves or $\mathscr{W}^{u}(\overline{\mathrm{x}})$ may not be a manifold. The following result gives a description of the stable and unstable sets of a saddle point of a competitive map. If the map is a diffeomorphism on $\mathscr{R}$, the sets $\mathscr{W}^{s}(\overline{\mathrm{x}})$ and $\mathscr{W}^{u}(\overline{\mathrm{x}})$ are the stable and unstable manifolds of $\bar{x}$.

Theorem 5. In addition to the hypotheses of part (B) of Theorem 4, suppose that $\mu>1$ and that the eigenspace $E^{\mu}$ associated with $\mu$ is not a coordinate axis. If the curve $\mathscr{C}$ of
Theorem 2 has endpoints in $\partial \mathscr{R}$, then $\mathscr{C}$ is the stable set $\mathscr{W}^{s}(\overline{\mathrm{x}})$ of $\overline{\mathrm{x}}$, and the unstable set $\mathscr{W}^{u}(\overline{\mathrm{x}})$ of $\bar{x}$ is a curve in $\mathscr{R}$ that is tangential to $E^{\mu}$ at $\overline{\mathrm{x}}$ and such that it is the graph of a strictly decreasing function of the first coordinate on an interval. Any endpoints of $\mathscr{W}^{u}(\overline{\mathrm{x}})$ in $\mathscr{R}$ are fixed points of $T$.

Remark 6. We say that $f(u, v)$ is strongly decreasing in the first argument and strongly increasing in the second argument if it is differentiable and has first partial derivative $D_{1} f$ negative and first partial derivative $D_{2} f$ positive in a considered set. The connection between the theory of monotone maps and the asymptotic behavior of (7) follows from the fact that if $f$ is strongly decreasing in the first argument and strongly increasing in the second argument, then the second iterate of a map associated to (7) is a strictly competitive map on $I \times I$; see [14].

Set $x_{n-1}=u_{n}$ and $x_{n}=v_{n}$ in (7) to obtain the equivalent system

$$
\begin{gathered}
u_{n+1}=v_{n} \\
v_{n+1}=f\left(v_{n}, u_{n}\right), \quad n=0,1, \ldots
\end{gathered}
$$

Let $T(u, v)=(v, f(v, u))$. The second iterate $T^{2}$ is given by

$$
T^{2}(u, v)=(f(v, u), f(f(v, u), v))
$$

and it is strictly competitive on $I \times I$ (see [14]).

Remark 7. The characteristic equation of (7) at an equilibrium point $(\bar{x}, \bar{x})$,

$$
\lambda^{2}-D_{1} f(\bar{x}, \bar{x}) \lambda-D_{2} f(\bar{x}, \bar{x})=0,
$$

has two real roots $\lambda$, $\mu$ which satisfy $\lambda<0<\mu$ and $|\lambda|<\mu$, whenever $f$ is strictly decreasing in first and increasing in second variable. Thus, the applicability of Theorems 2-5 depends on the nonexistence of minimal period-two solution.

There are several global attractivity results for (7). Some of these results give the sufficient conditions for all solutions to approach a unique equilibrium and they were used efficiently in [11]. The next result is from [17].

Theorem 8 (see [17]). Consider (7) where $f: I \times I \rightarrow I$ is a continuous function and $f$ is decreasing in the first argument and increasing in the second argument. Assume that $\bar{x}$ is a unique equilibrium point which is locally asymptotically stable and assume that $(\varphi, \psi)$ and $(\psi, \varphi)$ are minimal period-two solutions which are saddle points such that

$$
(\varphi, \psi) \preceq_{s e}(\bar{x}, \bar{x}) \preceq_{s e}(\psi, \varphi) .
$$

Then, the basin of attraction $\mathscr{B}((\bar{x}, \bar{x}))$ of $(\bar{x}, \bar{x})$ is the region between the global stable sets $\mathscr{W}^{\mathcal{s}}((\varphi, \psi))$ and $\mathscr{W}^{\mathcal{s}}((\psi, \varphi))$. More precisely,

$$
\begin{aligned}
& \mathscr{B}((\bar{x}, \bar{x}))=\left\{(x, y): \exists y_{u}, y_{l}: y_{u}<y<y_{l},\right. \\
&\left.\left(x, y_{l}\right) \in \mathscr{W}^{s}((\varphi, \psi)),\left(x, y_{u}\right) \in \mathscr{W}^{s}((\psi, \varphi))\right\} .
\end{aligned}
$$


The basins of attraction $\mathscr{B}((\varphi, \psi))=\mathscr{W}^{s}((\varphi, \psi))$ and $\mathscr{B}((\psi, \varphi))=\mathscr{W}^{s}((\psi, \varphi))$ are exactly the global stable sets of $(\varphi, \psi)$ and $(\psi, \varphi)$.

If $\left(x_{-1}, x_{0}\right) \in \mathscr{W}_{+}((\psi, \varphi))$ or $\left(x_{-1}, x_{0}\right) \in \mathscr{W}_{-}((\varphi, \psi))$, then $T^{n}\left(\left(x_{-1}, x_{0}\right)\right)$ converges to the other equilibrium point or to the other minimal period-two solutions or to the boundary of the region $I \times I$.

\section{Local Stability Analysis}

Denote

$$
f(u, v)=\frac{v^{2}}{a u^{2}+b u v+c v^{2}},
$$

and notice that the function $f(u, v)$ is decreasing in the first variable and increasing in the second variable. By Theorem 1, for every solutions $\left\{x_{n}\right\}_{n=-1}^{\infty}$ of (1) the subsequences $\left\{x_{2 n}\right\}_{n=0}^{\infty}$ and $\left\{x_{2 n-1}\right\}_{n=0}^{\infty}$ are eventually monotonic.

It is clear that (1) has a unique positive equilibrium solution $\bar{x}=1 /(a+b+c)$ and that a linearization of (1) is of the form

$$
y_{n+1}=s y_{n}+t y_{n-1}
$$

where

$$
\begin{aligned}
s & =-t=\frac{\partial f}{\partial u}(\bar{x}, \bar{x})=\left(-\frac{v^{2}(2 a u+b v)}{\left(a u^{2}+b u v+c v^{2}\right)^{2}}\right)(\bar{x}, \bar{x}) \\
& =-\frac{\bar{x}^{2}(2 a \bar{x}+b \bar{x})}{\left(a \bar{x}^{2}+b \bar{x}^{2}+c \bar{x}^{2}\right)^{2}}=-\frac{1}{\bar{x}} \cdot \frac{2 a+b}{(a+b+c)^{2}} \\
& =-\frac{2 a+b}{a+b+c} .
\end{aligned}
$$

Lemma 9. Equation (1) has a unique positive equilibrium solution $\bar{x}=1 /(a+b+c)$.

(i) If $3 a+b-c<0$, then equilibrium solution $\bar{x}$ is locally asymptotically stable.

(ii) If $3 a+b-c>0$, then equilibrium solution $\bar{x}$ is a saddle point.

(iii) If $3 a+b-c=0$, then equilibrium solution $\bar{x}$ is nonhyperbolic (with eigenvalues $\lambda_{1}=-1$ and $\lambda_{2}=1 / 2$ ).

Proof. By (17), a linearization of (1) is of the form

$$
y_{n+1}+\frac{2 a+b}{a+b+c} y_{n}-\frac{2 a+b}{a+b+c} y_{n-1}=0 .
$$

Its characteristic equation is

$$
\lambda^{2}+\frac{2 a+b}{a+b+c} \lambda-\frac{2 a+b}{a+b+c}=0
$$

with eigenvalues $\lambda_{ \pm}=(-(2 a+b) \pm \sqrt{D}) / 2(a+b+c)$, where $D=(2 a+b)(6 a+5 b+4 c)$. It is clear that $\lambda_{-}<$ 0 and $\lambda_{+}>0$. Now, we prove that $\lambda_{+} \in(0,1)$ and

$$
\lambda_{-} \begin{cases}\epsilon(-1,0) & \text { for } 3 a+b-c<0 \\ <-1 & \text { for } 3 a+b-c>0 \\ =-1 & \text { for } 3 a+b-c=0 .\end{cases}
$$

Namely,

(i) $\lambda_{+}<1 \Leftrightarrow-(2 a+b)+\sqrt{D}<2(a+b+c) \Leftrightarrow \sqrt{D}<$ $4 a+3 b+2 c \Leftrightarrow 4(a+b+c)^{2}>0$, which is always satisfied;

(ii) also,

$$
\begin{aligned}
\lambda_{-}<-1 & \Longleftrightarrow-(2 a+b)-\sqrt{D}<-2(a+b+c) \\
& \Longleftrightarrow \sqrt{D}>b+2 c \Longleftrightarrow(2 a+b)^{2}>(a-c)^{2} \\
& \Longleftrightarrow(a+b+c)(3 a+b-c)>0 \\
& \Longleftrightarrow 3 a+b-c>0 ;
\end{aligned}
$$

(iii) $\lambda_{-}>-1 \Leftrightarrow 3 a+b-c<0$ and $\lambda_{-}=-1 \Leftrightarrow 3 a+b-c=$ 0 .

Also, if $3 a+b-c=0$, then $c=3 a+b$, and we have $\lambda_{+}=$ $1 / 2$.

\section{Periodic Solutions}

In this section, we present results for the existence of minimal period-two solutions of (7).

Theorem 10. (a) Equation (1) has the minimal period-two solution

$$
\left\{\ldots, 0, \frac{1}{c}, 0, \frac{1}{c}, \ldots\right\}
$$

for all positive values of parameters $a, b$, and $c$.

(b) If $3 a+b-c<0$, then (1) has the minimal period-two solution:

$$
\{\ldots, \varphi, \psi, \varphi, \psi, \ldots\}, \quad(\varphi \neq \psi, \varphi>0, \psi>0),
$$

where

$$
\varphi=\frac{1-\sqrt{D}}{2(c-a)}, \quad \psi=\frac{1+\sqrt{D}}{2(c-a)}, \quad D=\frac{3 a+b-c}{b-a-c}>0 .
$$

Proof. Suppose that there is a minimal period-two solution $\{\varphi, \psi, \varphi, \psi, \ldots\}$ of (1), where $\varphi$ and $\psi$ are distinct nonnegative real numbers such that $\varphi^{2}+\psi^{2} \neq 0$. Then, $\varphi, \psi$ satisfy

$$
\begin{aligned}
& \varphi=\frac{\varphi^{2}}{a \psi^{2}+b \psi \varphi+c \varphi^{2}}, \\
& \psi=\frac{\psi^{2}}{a \varphi^{2}+b \varphi \psi+c \psi^{2}},
\end{aligned}
$$


from which we obtain three cases:

$$
\begin{gathered}
\varphi=0, \\
a \varphi^{2}+b \varphi \psi+c \psi^{2}=\psi, \\
a \psi^{2}+b \varphi \psi+c \varphi^{2}=\varphi, \\
\psi=0, \\
a \psi^{2}+b \varphi \psi+c \varphi^{2}=\varphi, \\
a \varphi^{2}+b \varphi \psi+c \psi^{2}=\psi .
\end{gathered}
$$

Conclusion (a) follows from (27) and (28).

Subtracting (30) from (29), we have

$$
(\psi-\varphi)[(a-c)(\varphi-\psi)+1]=0,
$$

that is,

$$
\varphi+\psi=\frac{1}{c-a}
$$

for $a<c$. Substituting (32) in (29), we obtain

$$
\varphi^{2}-\frac{1}{c-a} \varphi+\frac{a}{(a-b+c)(c-a)^{2}}=0,
$$

from which

$$
\varphi_{ \pm}=\frac{1 \pm \sqrt{D}}{2(c-a)} .
$$

Equation (32) implies that

$$
\psi_{ \pm}=\frac{1}{c-a}-\varphi_{ \pm}=\varphi_{\mp} .
$$

It is clear that

$$
\begin{aligned}
D= & \frac{3 a+b-c}{b-a-c}>0 \\
& \Longleftrightarrow\{(3 a+b-c>0 \wedge b-a-c>0) \\
\quad \quad \quad \times \vee(3 a+b-c<0 \wedge b-a-c<0)\} & (b-a>c \vee 3 a+b<c) .
\end{aligned}
$$

If $b-a>c$, then

$$
\begin{aligned}
D & =1+\frac{4 a}{b-a-c}>1 \\
& \Longrightarrow\left(\varphi_{-}<0, \varphi_{+}>0\right) \\
& \Longrightarrow\left(\psi_{-}>0, \psi_{+}<0\right),
\end{aligned}
$$

which is a contradiction.

If $3 a+b<c$, then

$$
0<D=1+\frac{4 a}{b-a-c}<1 \Longrightarrow\left(\varphi_{ \pm}>0, \psi_{ \pm}>0\right)
$$

By using substitution

$$
\begin{gathered}
x_{n-1}=u_{n}, \\
x_{n}=v_{n},
\end{gathered}
$$

equation (1) becomes the system of equations

$$
\begin{gathered}
u_{n+1}=v_{n}, \\
v_{n+1}=\frac{u_{n}^{2}}{a v_{n}^{2}+b u_{n} v_{n}+c u_{n}^{2}} .
\end{gathered}
$$

The map $T$ corresponding to the system (40) is of the form

$$
T\left(\begin{array}{l}
u \\
v
\end{array}\right)=\left(\begin{array}{c}
v \\
g(u, v)
\end{array}\right)
$$

where $g(u, v)=u^{2} /\left(a v^{2}+b u v+c u^{2}\right)$. The second iteration of the map $T$ is

$$
T^{2}\left(\begin{array}{l}
u \\
v
\end{array}\right)=T\left(\begin{array}{c}
v \\
g(u, v)
\end{array}\right)=\left(\begin{array}{c}
g(u, v) \\
g(v, g(u, v))
\end{array}\right)=\left(\begin{array}{l}
F(u, v) \\
G(u, v)
\end{array}\right),
$$

where

$$
F(u, v)=g(u, v), G(u, v)=\frac{v^{2}}{a F^{2}(u, v)+b v F(u, v)+c v^{2}}
$$

and the map $T^{2}$ is competitive by Remark 7. The Jacobian matrix of the map $T^{2}$ is

$$
J_{T^{2}}\left(\begin{array}{l}
u \\
v
\end{array}\right)=\left(\begin{array}{ll}
\frac{\partial F}{\partial u} & \frac{\partial F}{\partial v} \\
\frac{\partial G}{\partial u} & \frac{\partial G}{\partial v}
\end{array}\right)
$$

Notice that periodic solutions $(0,1 / c),(1 / c, 0),(\varphi, \psi)$, and $(\psi, \varphi)$ of (1) are equilibrium points of the map $T^{2}$. 
Now, we have

$$
\begin{aligned}
& \frac{\partial F}{\partial u}=\frac{2 u\left(a v^{2}+b u v+c u^{2}\right)-u^{2}(b v+2 c u)}{\left(a v^{2}+b u v+c u^{2}\right)^{2}} \\
& =\frac{u v(2 a v+b u)}{\left(a v^{2}+b u v+c u^{2}\right)^{2}}, \\
& \frac{\partial F}{\partial v}=-\frac{u^{2}(2 a v+b u)}{\left(a v^{2}+b u v+c u^{2}\right)^{2}}, \\
& \frac{\partial G}{\partial u}=-\frac{v^{2}}{\left(a F^{2}(u, v)+b v F(u, v)+c v^{2}\right)^{2}} \\
& \cdot(2 a F(u, v)+b v) \frac{\partial F}{\partial u}, \\
& \frac{\partial G}{\partial v}=\left(2 v\left(a F^{2}(u, v)+b v F(u, v)+c v^{2}\right)\right. \\
& -v^{2}(2 a F(u, v)(\partial F / \partial v)+b F(u, v) \\
& +b v(\partial F / \partial v)+2 c v)) \\
& \times\left(a F^{2}(u, v)+b v F(u, v)+c v^{2}\right)^{-2} \\
& =(v(2 a F(u, v)+b v) F(u, v) \\
& \left.-(2 a F(u, v)+b v) v^{2}(\partial F / \partial v)\right) \\
& \times\left(a F^{2}(u, v)+b v F(u, v)+c v^{2}\right)^{-2} .
\end{aligned}
$$

Theorem 11. (i) The minimal period-two points

$$
\left(0, \frac{1}{c}\right), \quad\left(\frac{1}{c}, 0\right)
$$

are locally asymptotically stable.

(ii) If $a<c$ and $3 a+b-c<0$, then the minimal period-two points

$$
(\varphi, \psi), \quad(\psi, \varphi) \quad(\varphi \neq \psi, \varphi>0, \psi>0),
$$

where $\varphi$ and $\psi$ satisfy (25), are saddle points.

Proof. (i) Since $F(0,1 / c)=0$, for periodic point $(0,1 / c)$, we have

$$
\begin{gathered}
\frac{\partial F}{\partial u}\left(\begin{array}{c}
0 \\
\frac{1}{c}
\end{array}\right)=\frac{\partial F}{\partial v}\left(\begin{array}{c}
0 \\
\frac{1}{c}
\end{array}\right)=\frac{\partial G}{\partial u}\left(\begin{array}{l}
0 \\
\frac{1}{c}
\end{array}\right)=\frac{\partial G}{\partial v}\left(\begin{array}{l}
0 \\
\frac{1}{c}
\end{array}\right)=0, \\
J_{T^{2}}\left(\begin{array}{l}
0 \\
\frac{1}{c}
\end{array}\right)=\left(\begin{array}{ll}
0 & 0 \\
0 & 0
\end{array}\right)
\end{gathered}
$$

with eigenvalues $\lambda_{1,2}=0$, which implies that $(0,1 / c)$ is locally asymptotically stable.
Similarly, since $F(1 / c, 0)=1 / c$, for periodic solution $(1 / c, 0)$, we have

$$
J_{T^{2}}\left(\begin{array}{c}
1 \\
c \\
0
\end{array}\right)=\left(\begin{array}{cc}
0 & -\frac{b}{c} \\
0 & 0
\end{array}\right)
$$

with eigenvalues $\lambda_{1,2}=0$, which implies that $(0,1 / c)$ is locally asymptotically stable.

(ii) By (26), we have that

$$
\begin{gathered}
F(\varphi, \psi)=\varphi, \\
\frac{\partial F}{\partial u}\left(\begin{array}{c}
\varphi \\
\psi
\end{array}\right)=\psi\left(2 a \frac{\psi}{\varphi}+b\right), \\
\frac{\partial F}{\partial v}\left(\begin{array}{l}
\varphi \\
\psi
\end{array}\right)=-\varphi\left(2 a \frac{\psi}{\varphi}+b\right) .
\end{gathered}
$$

By (50) and (51), we obtain

$$
\begin{aligned}
\frac{\partial G}{\partial u}\left(\begin{array}{c}
\varphi \\
\psi
\end{array}\right)= & -\frac{\psi^{2}}{\left(a \varphi^{2}+b \psi \varphi+c \psi^{2}\right)^{2}} \\
& \cdot(2 a \varphi+b \psi) \frac{\partial F}{\partial u}\left(\begin{array}{c}
\varphi \\
\psi
\end{array}\right) \\
= & -\psi(2 a \varphi+b \psi)\left(2 a \frac{\psi}{\varphi}+b\right) ;
\end{aligned}
$$

that is,

$$
\frac{\partial G}{\partial u}\left(\begin{array}{c}
\varphi \\
\psi
\end{array}\right)=-\psi^{2}\left(2 a \frac{\varphi}{\psi}+b\right)\left(2 a \frac{\psi}{\varphi}+b\right) .
$$

Similarly, we have

$$
\begin{aligned}
\frac{\partial G}{\partial v}\left(\begin{array}{c}
\varphi \\
\psi
\end{array}\right) & =\frac{\psi(2 a \varphi+b \psi) \varphi-(2 a \varphi+b \psi) \psi^{2}(\partial F / \partial v)\left(\begin{array}{l}
\varphi \\
\psi
\end{array}\right)}{\left(a \varphi^{2}+b \psi \varphi+c \psi^{2}\right)^{2}} \\
& =\frac{\psi(2 a \varphi+b \psi) \varphi+(2 a \varphi+b \psi) \psi^{2}(2 a \psi+b \varphi)}{\psi^{2}}
\end{aligned}
$$

so that

$$
\frac{\partial G}{\partial \nu}\left(\begin{array}{l}
\varphi \\
\psi
\end{array}\right)=\left(2 a \frac{\varphi}{\psi}+b\right) \varphi+\left(2 a \frac{\varphi}{\psi}+b\right)\left(2 a \frac{\psi}{\varphi}+b\right) \varphi \psi .
$$

Now, we obtain that Jacobian matrix of the map $T^{2}$ at the point $(\varphi, \psi)$ is of the form

$$
J_{T^{2}}\left(\begin{array}{l}
\varphi \\
\psi
\end{array}\right)=\left(\begin{array}{cc}
\frac{\partial F}{\partial u}\left(\begin{array}{c}
\varphi \\
\psi
\end{array}\right) & \frac{\partial F}{\partial v}\left(\begin{array}{l}
\varphi \\
\psi
\end{array}\right) \\
\frac{\partial G}{\partial u}\left(\begin{array}{c}
\varphi \\
\psi
\end{array}\right) & \frac{\partial G}{\partial v}\left(\begin{array}{c}
\varphi \\
\psi
\end{array}\right)
\end{array}\right) .
$$

The corresponding characteristic equation is

$$
\lambda^{2}-p \lambda+q=0,
$$


where

$$
\begin{aligned}
p= & \frac{\partial F}{\partial u}\left(\begin{array}{l}
\varphi \\
\psi
\end{array}\right)+\frac{\partial G}{\partial v}\left(\begin{array}{l}
\varphi \\
\psi
\end{array}\right) \\
= & \psi\left(2 a \frac{\psi}{\varphi}+b\right)+\varphi\left(2 a \frac{\varphi}{\psi}+b \psi\right) \\
& +\varphi \psi\left(2 a \frac{\varphi}{\psi}+b\right)\left(2 a \frac{\psi}{\varphi}+b\right),
\end{aligned}
$$

that is,

$$
p=m+n+m n,
$$

where

$$
m=\psi\left(2 a \frac{\psi}{\varphi}+b\right), \quad n=\varphi\left(2 a \frac{\varphi}{\psi}+b\right) .
$$

Notice that

$$
\begin{array}{ccc}
\frac{\partial F}{\partial u}\left(\begin{array}{l}
\varphi \\
\psi
\end{array}\right)=m, & \frac{\partial F}{\partial v}\left(\begin{array}{l}
\varphi \\
\psi
\end{array}\right)=-\frac{\varphi}{\psi} m, \\
\frac{\partial G}{\partial u}\left(\begin{array}{l}
\varphi \\
\psi
\end{array}\right)=-\frac{\psi}{\varphi} m n, & \frac{\partial G}{\partial v}\left(\begin{array}{l}
\varphi \\
\psi
\end{array}\right)=n(1+m),
\end{array}
$$

so that

$$
q=\operatorname{det} J_{T^{2}}\left(\begin{array}{l}
\varphi \\
\psi
\end{array}\right)=m n(1+m)-m^{2} n=m n .
$$

We need to show that

$$
|p|>|1+q| \quad p^{2}-4 q>0 .
$$

(i) Consider that

$$
\begin{aligned}
p^{2}-4 q>0 & \Longleftrightarrow(m+n+m n)^{2}-4 m n>0 \\
& \Longleftrightarrow m^{2}+n^{2}+m^{2} n^{2}+2 m^{2} n+2 m n^{2}>2 m n,
\end{aligned}
$$

which is satisfied because $m^{2}+n^{2} \geq 2 m n$.

(ii) Notice that

$$
\begin{aligned}
& m=\psi\left(2 a \frac{\psi}{\varphi}+b\right)=2 a \frac{\psi^{2}}{\varphi}+b \psi \\
& \stackrel{(26)}{=} \frac{2}{\varphi}\left(\varphi-b \varphi \psi-c \varphi^{2}\right)+b \psi=2-b \psi-2 c \varphi \\
& n=\varphi\left(2 a \frac{\varphi}{\psi}+b\right)=2 a \frac{\varphi^{2}}{\psi}+b \varphi \\
& \stackrel{(26)}{=} \frac{2}{\psi}\left(\psi-b \varphi \psi-c \psi^{2}\right)+b \varphi=2-b \varphi-2 c \varphi .
\end{aligned}
$$

This implies that

$$
\begin{aligned}
|p|>|1+q| & \Longleftrightarrow p>1+q \Longleftrightarrow m+n>1 \\
& \Longleftrightarrow 3-(b+c)(\varphi+\psi)>0 \\
& \stackrel{(18)}{\Leftrightarrow} 3-\frac{b+c}{c-a}>0 \Longleftrightarrow 3 a+b-c<0,
\end{aligned}
$$

which is satisfied.

\section{Global Results and Basins of Attraction}

In this section, we present global dynamics results for (1).

Notice that $T(u, 0)=(0,1 / c), u>0$, and $T(0, v)=$ $(v, 0), v>0$.

Theorem 12. If $3 a+b-c<0$, then (7) has a unique equilibrium point $\bar{x}$, which is locally asymptotically stable, and has the minimal period-two solution $(\varphi, \psi),(\psi, \varphi)$, which is a saddle point and has the minimal period-two solution $(0,1 / c)$, $(1 / c, 0)$ which is locally asymptotically stable. The basin of attraction $\mathscr{B}((\bar{x}, \bar{x}))$ of $(\bar{x}, \bar{x})$ is the region between the global stable sets $\mathscr{W}^{s}((\varphi, \psi))$ and $\mathscr{W}^{s}((\psi, \varphi))$. The basins of attraction $\mathscr{B}((\varphi, \psi))=\mathscr{W}^{s}((\varphi, \psi))$ and $\mathscr{B}((\psi, \varphi))=\mathscr{W}^{s}((\psi, \varphi))$ are exactly the global stable sets of $(\varphi, \psi)$ and $(\psi, \varphi)$. Furthermore, the basin of attraction of the minimal period-two solution $(0,1 / c),(1 / c, 0)$ is the union of the regions above $\mathscr{W}^{s}((\varphi, \psi))$ and below $\mathscr{W}^{s}((\psi, \varphi))$ in SE ordering; that is,

(i) if $\left(x_{-1}, x_{0}\right) \in \mathscr{W}_{-}((\varphi, \psi))$, then $\lim _{n \rightarrow \infty} x_{2 n}=$ $1 / c$ and $\lim _{n \rightarrow \infty} x_{2 n+1}=0$;

(ii) if $\left(x_{-1}, x_{0}\right) \in \mathscr{W}_{+}(\psi, \varphi)$, then $\lim _{n \rightarrow \infty} x_{2 n}=$ 0 and $\lim _{n \rightarrow \infty} x_{2 n+1}=1 / c$.

Proof. Using assumption $3 a+b-c<0$ (and its consequences: $c-a>0, b-c-a<0)$ and (25), it is easy to check that $(0,1 / c) \unlhd_{\text {se }}(\varphi, \psi) \unlhd_{\text {se }}(\bar{x}, \bar{x}) \preceq_{\text {se }}(\psi, \varphi) \unlhd_{\text {se }}(1 / c, 0)$. It is easy to check that the equilibrium point $(\bar{x}, \bar{x})$ is locally asymptotically stable for the strictly competitive map $T^{2}$ as well. Equation (1) is equivalent to the system of difference equations (40) which can be decomposed into the system of the evenindexed and odd-indexed terms as follows:

$$
\begin{gathered}
u_{2 n}=v_{2 n-1}, \\
u_{2 n+1}=v_{2 n}, \\
v_{2 n}=\frac{v_{2 n-1}^{2}}{a u_{2 n-1}^{2}+b u_{2 n-1} v_{2 n-1}+c v_{2 n-1}^{2}}, \\
v_{2 n+1}=\frac{v_{2 n}^{2}}{a u_{2 n}^{2}+b u_{2 n} v_{2 n}+c v_{2 n}^{2}} .
\end{gathered}
$$

The conclusion follows from Lemma 9 and from Theorems 10, 11, and 8 and using the facts that

(i) if $\left(x_{-1}, x_{0}\right) \in \mathscr{W}_{-}((\varphi, \psi))$, then

$$
\begin{gathered}
\left(u_{2 n}, v_{2 n}\right)=T^{2 n}\left(\left(u_{0}, v_{0}\right)\right) \longrightarrow\left(0, \frac{1}{c}\right), \\
\left(u_{2 n+1}, v_{2 n+1}\right)=T^{2 n+1}\left(\left(u_{0}, v_{0}\right)\right) \longrightarrow\left(\frac{1}{c}, 0\right) ;
\end{gathered}
$$




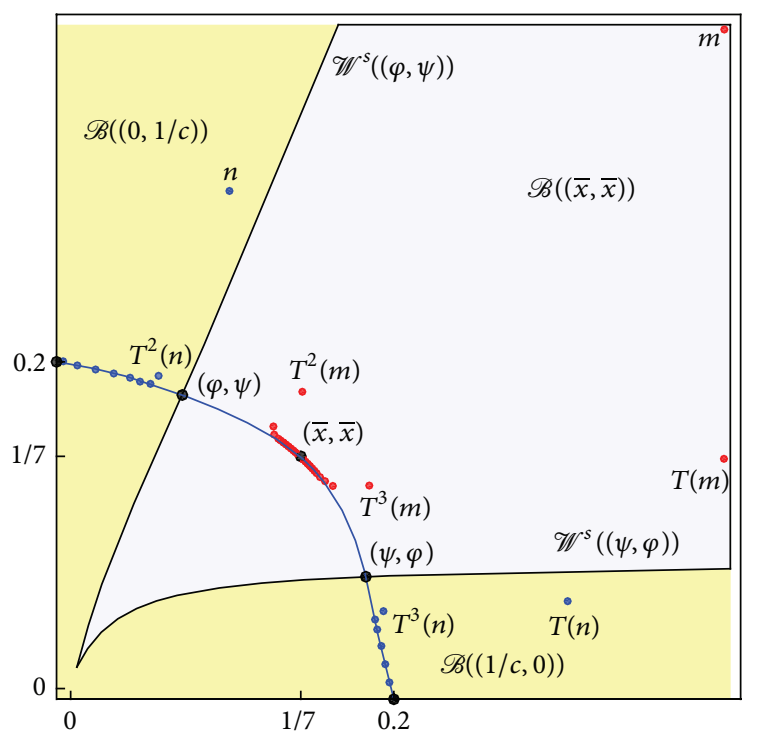

FIgURe 1: Global dynamics of (1) for $a=1, b=1$, and $c=5$-an illustration of Theorem 12.

(ii) if $\left(u_{0}, v_{0}\right) \in \mathscr{W}_{+}((\bar{x}, \bar{x}))$, then

$$
\begin{gathered}
\left(u_{2 n}, v_{2 n}\right)=T^{2 n}\left(\left(u_{0}, v_{0}\right)\right) \longrightarrow\left(\frac{1}{c}, 0\right), \\
\left(u_{2 n+1}, v_{2 n+1}\right)=T^{2 n+1}\left(\left(u_{0}, v_{0}\right)\right) \longrightarrow\left(0, \frac{1}{c}\right) .
\end{gathered}
$$

It means that

(i) if $\left(x_{-1}, x_{0}\right) \in \mathscr{W}_{-}((\bar{x}, \bar{x}))$, then $T^{2 n}\left(\left(x_{-1}, x_{0}\right)\right) \rightarrow$ $(0,1 / c)$ and $T^{2 n+1}\left(\left(x_{-1}, x_{0}\right)\right) \rightarrow(1 / c, 0)$, that is,

$$
\lim _{n \rightarrow \infty} x_{2 n}=\frac{1}{c} \quad \lim _{n \rightarrow \infty} x_{2 n+1}=0 ;
$$

(ii) if $\left(x_{-1}, x_{0}\right) \in \mathscr{W}_{+}(\psi, \varphi)$, then $T^{2 n+1}\left(\left(x_{-1}, x_{0}\right)\right) \rightarrow$ $(0,1 / c)$ and $T^{2 n}\left(\left(x_{-1}, x_{0}\right)\right) \rightarrow(1 / c, 0)$, that is,

$$
\lim _{n \rightarrow \infty} x_{2 n}=0 \quad \lim _{n \rightarrow \infty} x_{2 n+1}=\frac{1}{c}
$$

(see Figure 1).

Theorem 13. If $3 a+b-c>0$, then (1) has a unique equilibrium point $\bar{x}$ which is saddle point and has the minimal periodtwo solution $(0,1 / c),(1 / c, 0)$ which is locally asymptotically stable. There exists a set $\mathscr{C}$ which is an invariant subset of the basin of attraction of $(\bar{x}, \bar{x})$. The set $\mathscr{C}$ is a graph of a strictly increasing continuous function of the first variable on an interval and separates $\mathscr{R} \backslash(0,0)$, where $\mathscr{R}=[0, \infty) \times[0, \infty)$, into two connected and invariant components $\mathscr{W}_{-}((\bar{x}, \bar{x}))$ and $\mathscr{W}_{+}((\bar{x}, \bar{x}))$ which satisfy that

(i) if $\left(x_{-1}, x_{0}\right) \in \mathscr{W}_{-}((\bar{x}, \bar{x}))$, then $\lim _{n \rightarrow \infty} x_{2 n}=$ $1 / c$ and $\lim _{n \rightarrow \infty} x_{2 n+1}=0$;

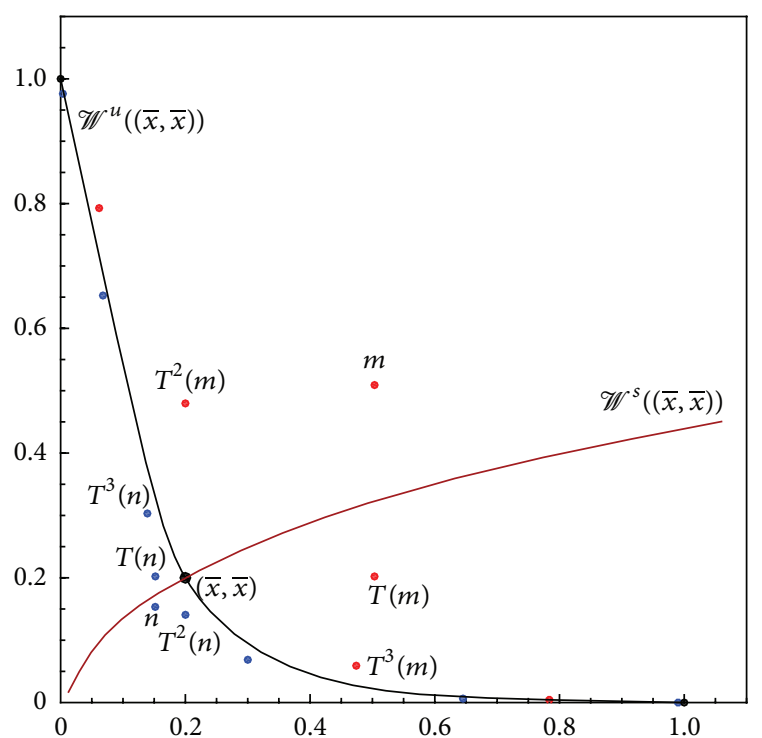

Figure 2: Global dynamics of (1) for $a=2, b=2$, and $c=1$-an illustration of Theorem 13.

(ii) if $\left(x_{-1}, x_{0}\right) \in \mathscr{W}_{+}((\bar{x}, \bar{x}))$, then $\lim _{n \rightarrow \infty} x_{2 n}=$ 0 and $\lim _{n \rightarrow \infty} x_{2 n+1}=1 / c$.

Proof. It is easy to check that $(\bar{x}, \bar{x})$ is a saddle point for the strictly competitive map $T^{2}$ as well. The existence of the set $\mathscr{C}$ with stated properties follows from Lemma 9 and Theorems 2,4 , and 10 . Therefore, using (68), we obtain that

(i) if $\left(u_{0}, v_{0}\right) \in \mathscr{W}_{-}((\bar{x}, \bar{x}))$,then

$$
\begin{gathered}
\left(u_{2 n}, v_{2 n}\right)=T^{2 n}\left(\left(u_{0}, v_{0}\right)\right) \longrightarrow\left(0, \frac{1}{c}\right), \\
\left(u_{2 n+1}, v_{2 n+1}\right)=T^{2 n+1}\left(\left(u_{0}, v_{0}\right)\right) \longrightarrow\left(\frac{1}{c}, 0\right) ;
\end{gathered}
$$

(ii) if $\left(u_{0}, v_{0}\right) \in \mathscr{W}_{+}((\bar{x}, \bar{x}))$, then

$$
\begin{gathered}
\left(u_{2 n}, v_{2 n}\right)=T^{2 n}\left(\left(u_{0}, v_{0}\right)\right) \longrightarrow\left(\frac{1}{c}, 0\right), \\
\left(u_{2 n+1}, v_{2 n+1}\right)=T^{2 n+1}\left(\left(u_{0}, v_{0}\right)\right) \longrightarrow\left(0, \frac{1}{c}\right) .
\end{gathered}
$$

Consequently,

(i) if $\left(x_{-1}, x_{0}\right) \in \mathscr{W}_{-}((\bar{x}, \bar{x}))$, then $T^{2 n}\left(\left(x_{-1}, x_{0}\right)\right) \rightarrow$ $(0,1 / c)$ and $T^{2 n+1}\left(\left(x_{-1}, x_{0}\right)\right) \rightarrow(1 / c, 0)$; that is,

$$
\lim _{n \rightarrow \infty} x_{2 n}=\frac{1}{c} \quad \lim _{n \rightarrow \infty} x_{2 n+1}=0 ;
$$

(ii) if $\left(x_{-1}, x_{0}\right) \in \mathscr{W}_{+}((\bar{x}, \bar{x}))$, then $T^{2 n+1}\left(\left(x_{-1}, x_{0}\right)\right) \rightarrow$ $(0,1 / c)$ and $T^{2 n}\left(\left(x_{-1}, x_{0}\right)\right) \rightarrow(1 / c, 0)$; that is,

$$
\lim _{n \rightarrow \infty} x_{2 n}=0 \quad \lim _{n \rightarrow \infty} x_{2 n+1}=\frac{1}{c}
$$

(see Figure 2). 


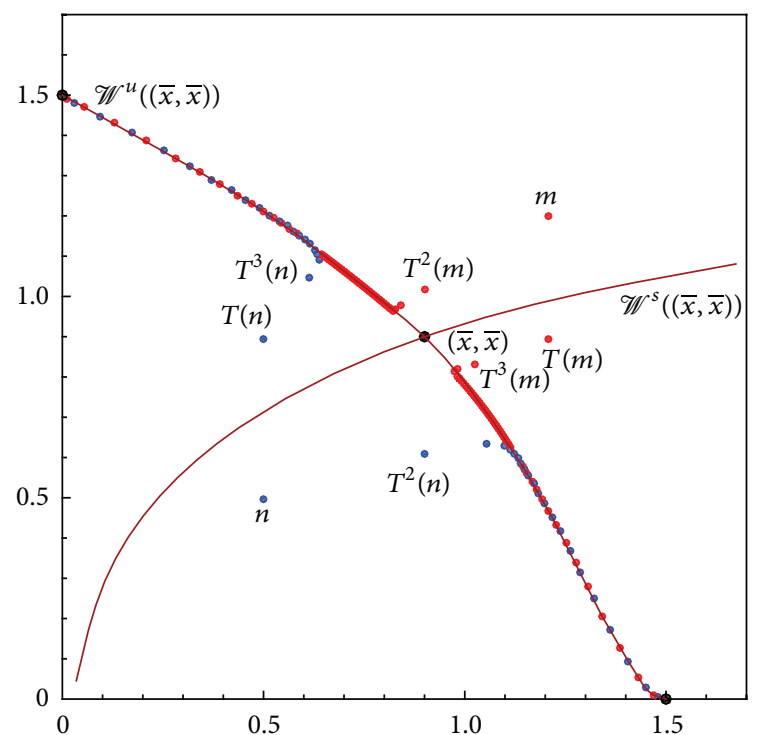

FIGURE 3: Global dynamics of (1) for $a=1 / 9, b=1 / 3$, and $c=$ 2/3-an illustration of Theorem 14.

Theorem 14. If $3 a+b-c=0$, then (1) has a unique equilibrium point $\bar{x}$ which is nonhyperbolic and has two minimal periodtwo points $(0,1 / c),(1 / c, 0)$ which are locally asymptotically stable points. There exists a set $\mathscr{C}$ which is an invariant subset of the basin of attraction of $(\bar{x}, \bar{x})$. The set $\mathscr{C}$ is a graph of a strictly increasing continuous function of the first variable on an interval and separates $\mathscr{R} \backslash(0,0)$, where $\mathscr{R}=[0, \infty) \times[0, \infty)$, into two connected and invariant components $\mathscr{W}_{-}((\bar{x}, \bar{x}))$ and $\mathscr{W}_{+}((\bar{x}, \bar{x}))$ which satisfy that

(i) if $\left(x_{-1}, x_{0}\right) \in \mathscr{W}_{-}((\bar{x}, \bar{x}))$, then $\lim _{n \rightarrow \infty} x_{2 n}=$ $1 / c$ and $\lim _{n \rightarrow \infty} x_{2 n+1}=0$;

(ii) if $\left(x_{-1}, x_{0}\right) \in \mathscr{W}_{+}((\bar{x}, \bar{x})), \lim _{n \rightarrow \infty} x_{2 n} \quad=$ 0 and $\lim _{n \rightarrow \infty} x_{2 n+1}=1 / c$.

Proof. In view of Lemma 9, the eigenvalues of the map $T$ at the equilibrium point $(\bar{x}, \bar{x})$ are $\lambda_{1}=-1$ and $\lambda_{2}=1 / 2$, which means that $\mu_{1}=\lambda_{1}^{2}=1$ and $\mu_{2}=\lambda_{2}^{2}=1 / 4$ are the eigenvalues of the map $T^{2}$. Using (51), (52), (54), and (56), we obtain

$$
J_{T^{2}}(\bar{x}, \bar{x})=\left(\begin{array}{cc}
Q & -Q \\
-Q^{2} & (Q-1)(Q-2)
\end{array}\right),
$$

where $Q=(2 a+b) /(a+b+c)$. A straightforward calculation yields that the eigenvector corresponding to the eigenvalue $\mu_{2}=1 / 4$ is of the form

$$
\mathbf{v}_{\mathbf{2}}=\left(\begin{array}{c}
2 \alpha \\
\alpha
\end{array}\right), \quad \alpha \in \mathbb{R} \backslash\{0\} .
$$

We see that eigenvector $\mathbf{v}_{2}$ is not parallel to coordinate axes. Therefore, all conditions of Theorem 2 are satisfied for the map $T^{2}$ with $\mathscr{R}=(0, \infty) \times(0, \infty)$. As a consequence of this and using (68), we have that

(i) if $\left(u_{0}, v_{0}\right) \quad \in \quad \mathscr{W}_{-}((\bar{x}, \bar{x}))$, then $\left(u_{2 n}, v_{2 n}\right) \quad \rightarrow$ $(0,1 / c)$ and $\left(u_{2 n+1}, v_{2 n+1}\right) \rightarrow(1 / c, 0)$; (ii) if $\left(u_{0}, v_{0}\right) \in \mathscr{W}_{+}((\bar{x}, \bar{x}))$, then $\left(u_{2 n}, v_{2 n}\right) \rightarrow$ $(1 / c, 0)$ and $\left(u_{2 n+1}, v_{2 n+1}\right) \rightarrow(0,1 / c)$.

It means that

(i) if $\left(x_{-1}, x_{0}\right) \in \mathscr{W}_{-}((\bar{x}, \bar{x}))$, then $T^{2 n}\left(\left(x_{-1}, x_{0}\right)\right) \rightarrow$ $(0,1 / c)$ and $T^{2 n+1}\left(\left(x_{-1}, x_{0}\right)\right) \rightarrow(1 / c, 0)$; that is,

$$
\lim _{n \rightarrow \infty} x_{2 n}=\frac{1}{c} \quad \lim _{n \rightarrow \infty} x_{2 n+1}=0 ;
$$

(ii) if $\left(x_{-1}, x_{0}\right) \in \mathscr{W}_{+}((\bar{x}, \bar{x}))$, then $T^{2 n+1}\left(\left(x_{-1}, x_{0}\right)\right) \rightarrow$ $(0,1 / c)$ and $T^{2 n}\left(\left(x_{-1}, x_{0}\right)\right) \rightarrow(1 / c, 0)$; that is,

$$
\lim _{n \rightarrow \infty} x_{2 n}=0 \quad \lim _{n \rightarrow \infty} x_{2 n+1}=\frac{1}{c}
$$

(see Figure 3).

Remark 15. As one may notice from the figures all stable manifolds of either saddle point equilibrium points or saddle period-two solutions are asymptotic to the origin, which is the point where (1) is not defined. These manifolds cannot end in any other point on the axes since the union of axes without the origin is an invariant set. Thus, the limiting points of the global stable manifolds of either saddle point equilibrium points or saddle period-two solutions have endpoints at $(0,0)$ and $(\infty, \infty)$.

\section{References}

[1] M. Dehghan, C. M. Kent, R. Mazrooei-Sebdani, N. L. Ortiz, and H. Sedaghat, "Dynamics of rational difference equations containing quadratic terms," Journal of Difference Equations and Applications, vol. 14, no. 2, pp. 191-208, 2008.

[2] M. Dehghan, C. M. Kent, R. Mazrooei-Sebdani, N. L. Ortiz, and H. Sedaghat, "Monotone and oscillatory solutions of a rational difference equation containing quadratic terms," Journal of Difference Equations and Applications, vol. 14, no. 10-11, pp. 1045-1058, 2008.

[3] C. M. Kent and H. Sedaghat, "Global attractivity in a quadraticlinear rational difference equation with delay," Journal of Difference Equations and Applications, vol. 15, no. 10, pp. 913-925, 2009.

[4] C. M. Kent and H. Sedaghat, "Global attractivity in a rational delay difference equation with quadratic terms," Journal of Difference Equations and Applications, vol. 17, no. 4, pp. 457-466, 2011.

[5] H. L. Smith, "Planar competitive and cooperative difference equations," Journal of Difference Equations and Applications, vol. 3, no. 5-6, pp. 335-357, 1998.

[6] E. Drymonis and G. Ladas, "On the global character of the rational system $x_{n+1}=\alpha_{1} / A_{1}+B_{1} x_{n}+y_{n}$ and $y_{n}+1=$ $\alpha_{2}+\beta_{2} x_{n} / A_{2}+B_{2} x_{n}+C_{2} y_{n}$ "' Sarajevo Journals of Mathematics, vol. 8, no. 21, pp. 293-309, 2012.

[7] M. Garić-Demirović, M. R. S. Kulenović, and M. Nurkanović, "Global behavior of four competitive rational systems of difference equations in the plane," Discrete Dynamics in Nature and Society, vol. 2009, Article ID 153058, 34 pages, 2009.

[8] E. A. Grove, D. Hadley, E. Lapierre, and S. W. Schultz, "On the global behavior of the rational system $x_{n}+1=\alpha_{1} / x_{n}+y_{n}$ and $y_{n}+1=\alpha_{2}+\beta_{2} x_{n}+y_{n} / y_{n}$," Sarajevo Journals of Mathematics, vol. 8, no. 21, pp. 283-292, 2012. 
[9] G. Ladas, G. Lugo, and F. J. Palladino, "Open problems and conjectures on rational systems in three dimensions," Sarajevo Journals of Mathematics, vol. 8, no. 21, pp. 311-321, 2012.

[10] S. Moranjkić and Z. Nurkanović, "Basins of attraction of certain rational anti-competitive system of difference equations in the plane," Advances in Difference Equations, vol. 2012, article 153, 2012.

[11] M. R. S. Kulenović and G. Ladas, Dynamics of Second Order Rational Difference Equations with Open Problems and Conjectures, Chapman and Hall/CRC, Boca Raton, Fla, USA, 2001.

[12] H. Sedaghat, "Global behaviours of rational difference equations of orders two and three with quadratic terms," Journal of Difference Equations and Applications, vol. 15, no. 3, pp. 215-224, 2009.

[13] O. Merino and M. R. S. Kulenović, "Global bifurcation for discrete competitive systems in the plane," Discrete and Continuous Dynamical Systems B, vol. 12, no. 1, pp. 133-149, 2009.

[14] M. R. S. Kulenović and O. Merino, "Invariant manifolds for competitive discrete systems in the plane," International Journal of Bifurcation and Chaos, vol. 20, no. 8, pp. 2471-2486, 2010.

[15] E. Camouzis and G. Ladas, "When does local asymptotic stability imply global attractivity in rational equations?" Journal of Difference Equations and Applications, vol. 12, no. 8, pp. 863885, 2006.

[16] H. L. Smith, "Periodic competitive differential equations and the discrete dynamics of competitive maps," Journal of Differential Equations, vol. 64, no. 2, pp. 165-194, 1986.

[17] M. Garić-Demirović, M. R. S. Kulenović, and M. Nurkanović, "Basins of attraction of equilibrium points of second order difference equations," Applied Mathematics Letters, vol. 25, pp. 2110-2115, 2012. 


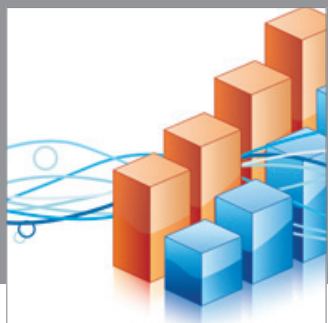

Advances in

Operations Research

mansans

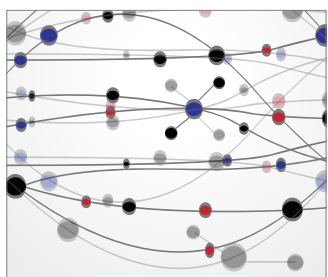

The Scientific World Journal
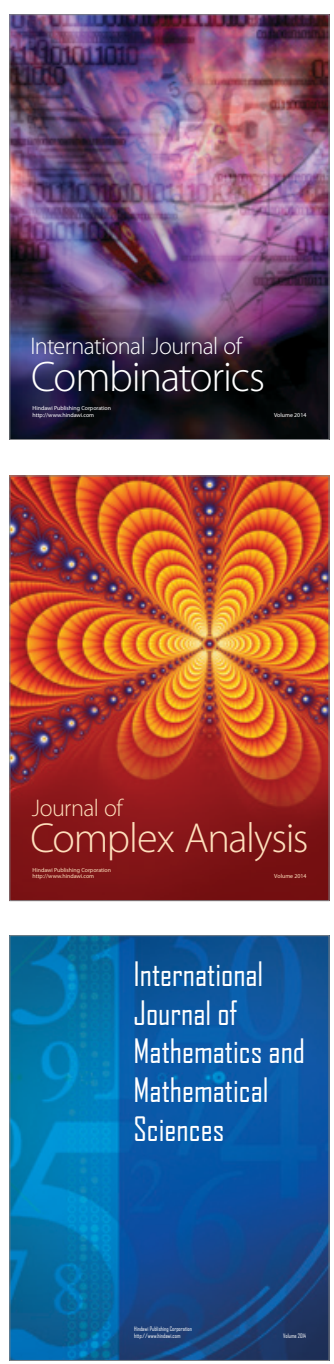
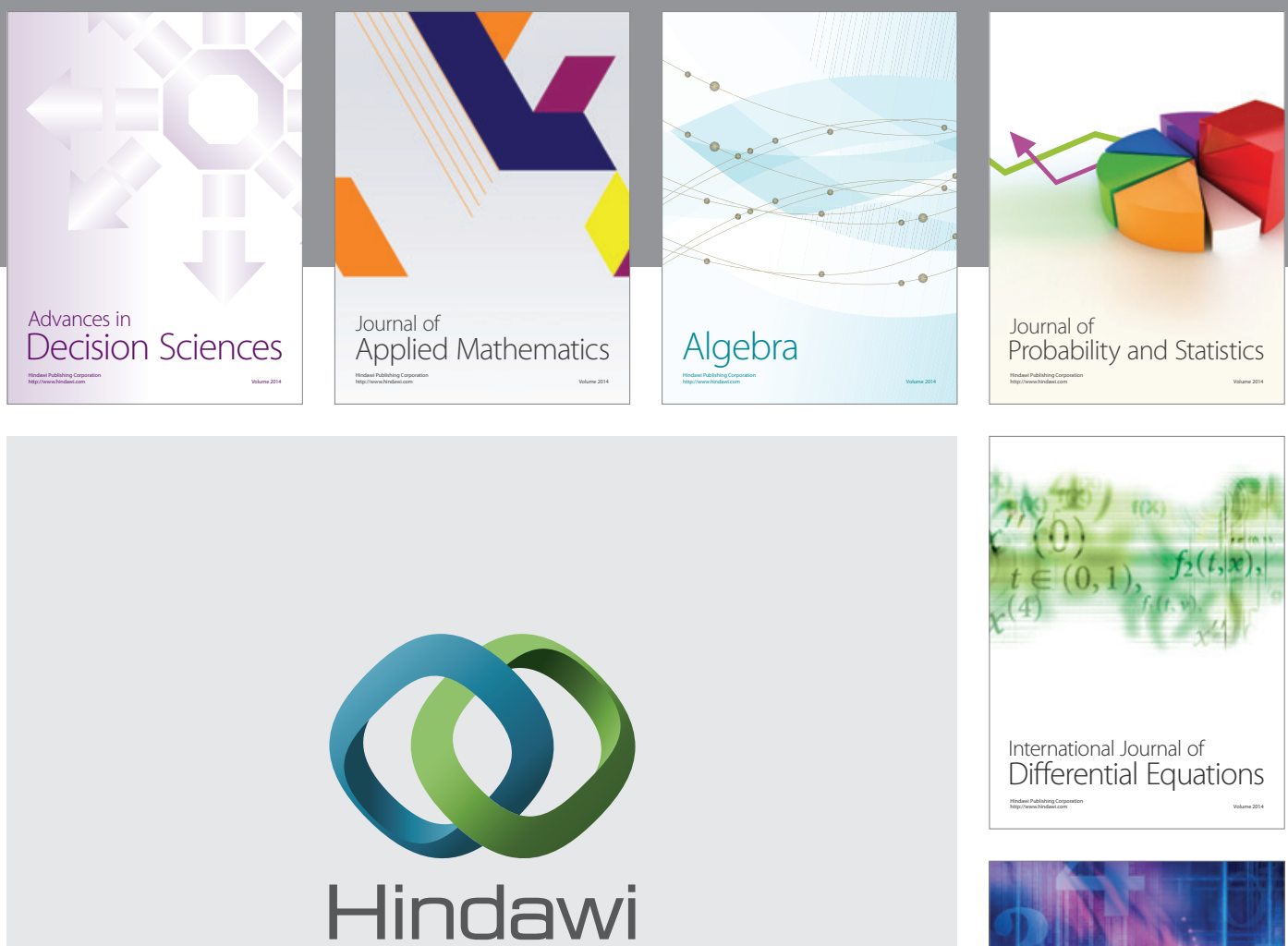

Submit your manuscripts at http://www.hindawi.com
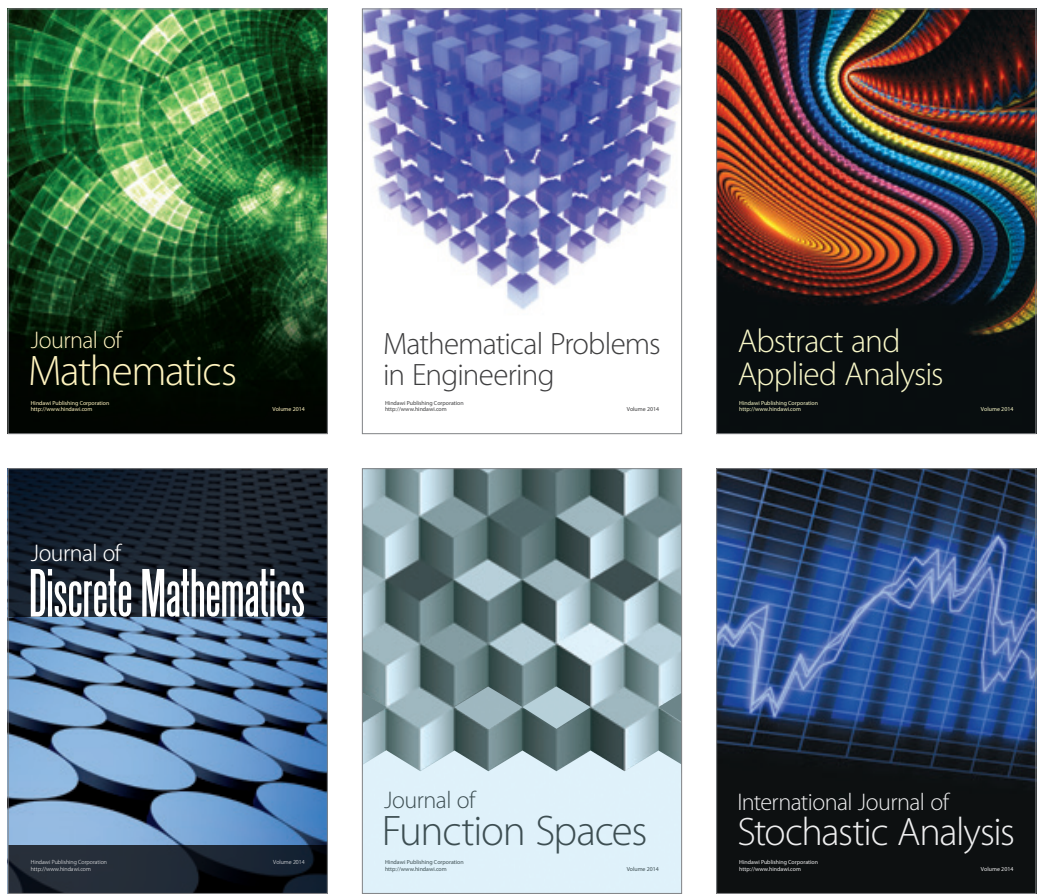

Journal of

Function Spaces

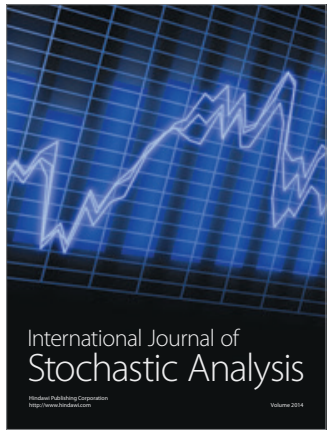

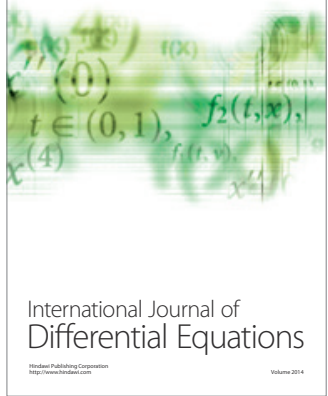
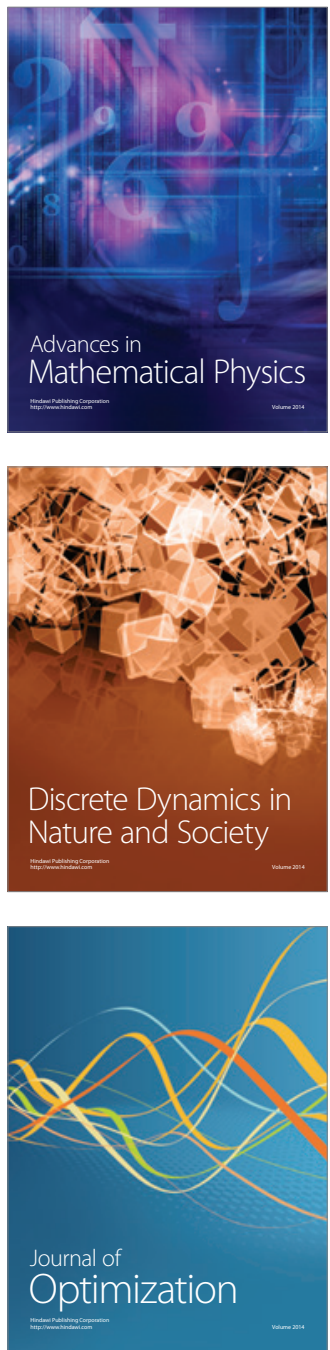